\title{
On the Radio-Frequency Inputs in Dipolar Heating of Adhesives *
}

\author{
H.T. Banks \\ Center for Research in Scientific Computation \\ Box 8205 North Carolina State University \\ Raleigh, NC 27695-8205
}

S.R. Durso

Lord Corporation

Thomas Lord Research Center

110 Lord Drive P.O. Box 8012

Cary, NC 27512-8012
M.A. Goodhart and M.L. Joyner
Center for Research in Scientific Computation
Box 8205 North Carolina State University
Raleigh, NC 27695-8205

March 19, 1999

${ }^{*}$ Research supported in part by the US Air Force Office of Scientific Research under grants AFOSR F-49620-95-1-0236 and AFOSR F-49620-95-1-0375 and by the US Department of Education under grant P200A40730. 


\begin{abstract}
We consider the form of the radio-frequency $(\mathrm{RF})$ or dielectric input expression in the heat equation which arises in the modeling of the curing of epoxy adhesives in bonding of composites. We review two standard derivations of a commonly used expression for the RF heating source term. In this context we discuss difficulties involving the associated inherent polarization assumption and asymptotic behavior of dielectric parameters as a function of the frequency of the RF signal. These difficulties cast doubt on the validity of the standard RF expression. We then use two standard polarization models (Debye and Lorentz) to demonstrate how one can systematically derive general RF heating expressions which do not suffer the inconsistencies that arise in the standard RF expression.
\end{abstract}




\section{Introduction}

The use of radio-frequency $(\mathrm{RF})$ electromagnetic energy for the curing of adhesives is a commercially important process. The complexity of this process, one involving phase transitions and exothermic chemical reactions, provides a number of interesting scientific and technical questions and challenges. The application of interest involves the adhesive bonding of sheet molding compound (SMC), a glass fiber reinforced polyester commonly used in the automotive industry for exterior body panels. In this process, a thin layer of nonmagnetic adhesive is placed between two sheets of SMC. This adhesive "sandwich" is placed between two aluminum electrodes which contact the part. A high voltage (e.g., 1-10 KV) electric field is applied across the part at $30 \mathrm{MHz}$. The field passes through the sheet of SMC, which is relatively non-dielectric (i.e., a conductor), and into the dielectric adhesive. The adhesive heats and eventually cures to bond the sheets of SMC together. One interesting computational challenge is to model and predict the heating of adhesives so that precise control algorithms can be developed for the production process. We have in [Banks, et. al., 1998] developed such a model along with an efficient computational simulation method.

The model used to describe the heating of the adhesive is a 3 -dimensional nonlinear heat equation [Bejan, 1993, p. 16], [Malaczynski and Cipinski, 1996, p. 107]

$$
\rho c_{p} \frac{\partial T}{\partial t}=\nabla \cdot(\kappa \cdot \nabla T)+\dot{q}_{e x}+\dot{q}_{r f}
$$

where $\rho$ is mass density, $c_{p}$ is specific heat, $\rho c_{p}$ is volumetric heat capacity, and $\kappa$ is thermal conductivity of the adhesive which, of course, may all be dependent on $T$ (e.g., see [Banks, et. al., 1998]). The term $\dot{q}_{e x}$ represents the rate of heating due to an internal exothermic reaction and $\dot{q}_{r f}$ is the rate of heat generated from conversion of electrical energy to molecular vibrational energy. (Here the overdots, which are standard notation, represent rates, not actual time derivatives). Our discussions here focus on the form of the $\dot{q}_{r f}$ term. Other aspects of the model, including a detailed explanation of the exothermic term $\dot{q}_{e x}$, which depends on the degree of cure and temperature in a nonlinear manner, are discussed in the paper [Banks, et. al., 1998].

In this note we address issues related to the RF input term $\dot{q}_{r f}$ and the validity of a commonly used 
form of this term. In our discussions we offer possible alternatives to the usual expression found in the research literature along with suggestions as to why these alternatives might be more appropriate in certain applications.

\section{Review of derivation of standard RF term}

In investigating the term $\dot{q}_{r f}$, one encounters the following form which is widely used in literature [Bunget and Popescu, 1984, p. 17], [Frohlich, 1958, p. 14], [Malaczynski, 1988, p. 1271],

[Malaczynski and Cipinski, 1996, p. 107], [Ross, 1982, p. 5824], [Scaife, 1989, p. 13], [White, 1973, p. 48]:

$$
\dot{q}=\omega \varepsilon_{0} \varepsilon_{R}^{\prime} \tan \delta E_{R M S}^{2} .
$$

Here $\omega$ is the frequency of the RF bonder, $\varepsilon_{0}$ is the permittivity of free space, $\varepsilon_{R}^{\prime}=\frac{\varepsilon^{\prime}}{\varepsilon_{0}}$ is the relative dielectric constant where $\varepsilon=\varepsilon^{\prime}-i \varepsilon^{\prime \prime}$ is the complex permittivity, $\tan \delta=\frac{\varepsilon^{\prime \prime}}{\varepsilon^{\prime}}$ is the dielectric loss tangent, and $E_{R M S}$ is the root mean square strength of the electric field being applied to the part. Here we consider first the method found in [Frohlich, 1958] to derive (1).

\subsection{Frohlich's Derivation}

Much of the literature tacitly assumes that dielectric heating is accomplished through dipolar rotation and we shall make this assumption for the present. This means that energy dispersion/conversion via polarization is of foremost importance. A first step in deriving (1) is understanding the assumptions which lead to the definition $\tan \delta=\frac{\varepsilon^{\prime \prime}}{\varepsilon^{\prime}}$. Following Frohlich, we consider the case in which one applies a periodic electric field $E=E_{0} \cos \omega t$ where $E_{0}$, the magnitude of the electric field, is independent of time and $\omega / 2 \pi$ is frequency in cycles per second. In this note we will denote the electric flux density (or displacement) by $D=\varepsilon_{0} \varepsilon_{\infty} E+P$ where $P$ is the polarization and $\varepsilon_{\infty}$ is the upper limit for $\varepsilon$ as $\omega \rightarrow \infty$ [Frohlich, 1958, p. 5]. This differs from much of the literature where the convention is $D=\varepsilon_{0} E+P$, but this change is necessary to ensure that the time domain polarization formulations are consistent with the frequency domain formulations often found in literature. Additionally, in some of the literature, derivations are carried out in the Gaussian system where $\varepsilon_{0}$ is not present in the definition of $D$. Since our considerations 
are carried out in the mks system they may differ slightly (by scale factors) from those. As

in [Frohlich, 1958], we assume that if $E$ is periodic in time, $P$ will also eventually be periodic in time. Then $D$ will also be periodic in time since $D=\varepsilon_{0} \varepsilon_{\infty} E+P$. (In general this is not true. For example, in a Debye or Lorentz medium, $\mathrm{P}$ is only asymptotic to $\mathrm{E}$.

In the Frohlich derivation one also assumes that while $D$ will be of the same period as $E$, it will not necessarily be in phase with $E$. The rationale is that matter does not respond instantaneously to excitement induced by electromagnetic waves, but will respond with some hysteresis. This phenomenon can be expressed by the widely used expression (e.g., see [Stuerga and Gaillard, 1996, p. 95])

$$
P(t)=\int_{t-r}^{t} K(t-s) E(s) d s
$$

which embodies the assumption that the polarization at time $t$ depends on the electric field over an immediate past period of length $r$. If we consider a two point quadrature (for example, a trapezoid rule for the integral -see [Burden and Faires, 1993, p. 176]) using the endpoints, from this polarization assumption we obtain an approximation of the form

$$
P(t) \approx K_{1} E(t)+K_{2} E(t-r)
$$

where $K_{1}=\frac{r}{2} K(0)$ and $K_{2}=\frac{r}{2} K(r)$. This means that $P$ depends on $E$ at a past time. Therefore there is a delay in $P$ and hence in $D$. This delay is represented by a phase shift $\delta$. Let $D_{0}$ denote the magnitude of the electric displacement $D$. Then

$$
\begin{aligned}
D & =D_{0} \cos (\omega t-\delta) \\
& =D_{0}(\cos \omega t \cos \delta+\sin \omega t \sin \delta) \\
& =\left(D_{0} \cos \delta\right) \cos \omega t+\left(D_{0} \sin \delta\right) \sin \omega t \\
D & =D_{1} \cos \omega t+D_{2} \sin \omega t
\end{aligned}
$$

where $D_{1}=D_{0} \cos \delta$ and $D_{2}=D_{0} \sin \delta$.

For most dielectrics, $D_{0}$ will be proportional to $E_{0}$ and $D_{0} / E_{0}$ will depend on factors such as frequency, amplitude, temperature and humidity. For the Frohlich derivations, however, one assumes that 
$\varepsilon^{\prime}$ and $\varepsilon^{\prime \prime}$ depend solely on frequency. We introduce $\varepsilon^{\prime}(\omega)$ and $\varepsilon^{\prime \prime}(\omega)$ as

$$
D_{1}=\varepsilon^{\prime} E_{0} \quad D_{2}=\varepsilon^{\prime \prime} E_{0},
$$

and note that the frequency dependence of $\varepsilon^{\prime}$ and $\varepsilon^{\prime \prime}$ is not directly an issue for our application to curing of adhesives since we work at only one frequency in our experiments. We have $D_{1}=\varepsilon^{\prime} E_{0}=D_{0} \cos \delta$ and $D_{2}=\varepsilon^{\prime \prime} E_{0}=D_{0} \sin \delta$ and consequently:

$$
\frac{\varepsilon^{\prime \prime}}{\varepsilon^{\prime}}=\frac{D_{0} \sin \delta}{D_{0} \cos \delta}=\tan \delta
$$

More generally, we note that for a general electric field $E(t)=E_{0} e^{i \omega t}$, the formulation from (2) and (3) is equivalent to

$$
\begin{aligned}
D & =D_{0} e^{i(\omega t-\delta)}=\left(D_{0} \cos \delta-i D_{0} \sin \delta\right) e^{i \omega t} \\
& =\left(\varepsilon^{\prime}-i \varepsilon^{\prime \prime}\right) E_{0} e^{i \omega t}=\varepsilon E
\end{aligned}
$$

where $\varepsilon=\varepsilon^{\prime}-i \varepsilon^{\prime \prime}$ is the complex permittivity.

We continue the derivation of (1) using the equation:

$$
d U=d Q+E d D
$$

where $d U$ is the increase in total energy per unit volume, $d Q$ is the influx of heat per unit volume, and $E d D$ is the influx of electromagnetic energy into the dielectric per unit volume if the displacement $D$ is increased by $d D$ (see Section 2.1.1 below for an explanation of this term) possibly due to a change in the field strength $E$ at a constant temperature or to a change in temperature at a constant $E$ [Frohlich, 1958, p. 11]. We calculate the amount of electric energy transformed into heat on an average of one period. Since we assume that the total energy is kept constant and $E$ is periodic, $U$ must be constant and hence $d U=0$ in equation (4); this yields

$$
d Q=-E d D
$$

and hence over a period of $[0,2 \pi / \omega]$ we have

$$
\int_{0}^{2 \pi / \omega} d Q=-\int_{0}^{2 \pi / \omega} E d D=-\int_{0}^{2 \pi / \omega} E \frac{\partial D}{\partial t} d t
$$


Then the average (over one period) rate of loss $L$ of energy from the electric field (the heat produced per second per unit volume) is given by

$$
L=-\frac{1}{\frac{2 \pi}{\omega}} \int_{0}^{2 \pi / \omega} d Q=-\frac{\omega}{2 \pi} \int_{0}^{2 \pi / \omega} d Q=\frac{\omega}{2 \pi} \int_{0}^{2 \pi / \omega} E \frac{\partial D}{\partial t} d t
$$

We recall $E=E_{0} \cos \omega t$, and from (2) we have $D=\varepsilon^{\prime} E_{0} \cos \omega t+\varepsilon^{\prime \prime} E_{0} \sin \omega t$, and hence,

$$
\begin{aligned}
L & =\frac{\omega}{2 \pi} E_{0}^{2} \int_{0}^{2 \pi / \omega} \cos \omega t\left(\varepsilon^{\prime} \frac{\partial \cos \omega t}{\partial t}+\varepsilon^{\prime \prime} \frac{\partial \sin \omega t}{\partial t}\right) d t \\
& =\frac{\omega E_{0}^{2}}{2 \pi}\left(\int_{0}^{2 \pi / \omega} \frac{\varepsilon^{\prime}}{2} \frac{\partial}{\partial t}\left(\cos ^{2} \omega t\right) d t+\int_{0}^{2 \pi / \omega} \varepsilon^{\prime \prime} \omega \cos ^{2} \omega t d t\right) \\
& =\left.\frac{\omega E_{0}^{2}}{2 \pi} \frac{\varepsilon^{\prime}}{2} \cos ^{2} \omega t\right|_{0} ^{2 \pi / \omega}+\frac{\omega^{2} E_{0}^{2}}{2 \pi} \varepsilon^{\prime \prime}\left(\frac{\pi}{\omega}\right) \\
& =\frac{\varepsilon^{\prime \prime} E_{0}^{2} \omega}{2} .
\end{aligned}
$$

Since $\varepsilon^{\prime \prime}=\varepsilon^{\prime} \tan \delta$, we thus have

$$
\begin{aligned}
L & =\frac{1}{2} \varepsilon^{\prime}(\tan \delta) E_{0}^{2} \omega \\
& =\frac{1}{2} \varepsilon_{0} \frac{\varepsilon^{\prime}}{\varepsilon_{0}} \omega E_{0}^{2} \tan \delta \\
& =\frac{1}{2} \varepsilon_{0} \varepsilon_{R}^{\prime} \omega E_{0}^{2} \tan \delta
\end{aligned}
$$

If we let $E_{R M S}=E_{0} / \sqrt{2}$ then we have

$$
\dot{q}=L=\varepsilon_{0} \varepsilon_{R}^{\prime} \omega E_{R M S}^{2} \tan \delta
$$

which is exactly equation (1).

\subsubsection{Origin of $E d D$ term}

We return to consider the rationale behind using the term $E d D$ as we did in section 2.1 (e.g., the rationale behind (4)). We begin with Maxwell's Equations:

$$
\begin{aligned}
\nabla \times E & =-\frac{\partial B}{\partial t} \\
\nabla \times H & =\frac{\partial D}{\partial t}+J \\
\nabla \cdot D & =\rho \\
\nabla \cdot B & =0
\end{aligned}
$$


where $D=D(E)$ and $B=B(H)$. If we multiply (7) by $E,(6)$ by $H$ and subtract, we obtain

$$
E \cdot(\nabla \times H)-H \cdot(\nabla \times E)=E \cdot\left(\frac{\partial D}{\partial t}+J\right)+H \cdot\left(\frac{\partial B}{\partial t}\right)
$$

Using the identity $A \cdot(\nabla \times B)-B \cdot(\nabla \times A)=\nabla \cdot(B \times A)$ we write

$$
-\nabla \cdot(E \times H)=E \cdot\left(\frac{\partial D}{\partial t}+J\right)+H \cdot\left(\frac{\partial B}{\partial t}\right)
$$

which can be rewritten as

$$
\left(E \cdot \frac{\partial D}{\partial t}+H \cdot \frac{\partial B}{\partial t}\right)+\nabla \cdot(E \times H)+E \cdot J=0 .
$$

If we assume that $B=\mu_{0} H$,

$$
\underbrace{\left(E \cdot \frac{\partial D}{\partial t}+\mu_{0} H \cdot \frac{\partial H}{\partial t}\right)}_{(a)}+\underbrace{\nabla \cdot S}_{(b)}+\underbrace{E \cdot J}_{(c)}=0
$$

where $S=E \times H$ is the Poynting vector. The term (b) gives us the rate of efflux of E-M energy per unit volume. The term (c) gives us the rate of conversion of E-M energy into other types of energy per unit volume. Therefore, by conservation of energy, (a) must represent the rate of change of energy content per unit volume. We further note that

$$
\mu_{0} H \cdot \frac{\partial H}{\partial t}=\frac{\mu_{0}}{2} \frac{\partial}{\partial t}\left(H^{2}\right)=\frac{\partial}{\partial t} \frac{\mu_{0} H^{2}}{2}
$$

where $\frac{1}{2} \mu_{0} H^{2}$ is the density of magnetic energy. As a result it is usually suggested that $\int E(D) d D$ is the density of electric energy, which finally suggests that $E d D$ is the change of energy density (per unit volume) connected with a variation of $D$ by $d D$.

\subsection{Necessity for alternate derivations}

In studying the derivation above and the resulting expression (1), we encountered several problems with both the intuitive behavior of the RF term at high frequencies and with the implicit assumptions made about polarization underlying the derivation. We discuss these difficulties here to establish that there is a necessity for alternate derivations and interpretations for $\dot{q}_{r f}$.

In general we have $D=\varepsilon_{0} \varepsilon_{\infty} E+P=\varepsilon E$ or in frequency domain $\hat{D}(\omega)=\varepsilon(\omega) \hat{E}(\omega)$ where $\hat{D}$ and $\hat{E}$ denote the Laplace transforms of $D$ and $E$, respectively and the complex permittivity is 
$\varepsilon(\omega)=\varepsilon_{r e}(\omega)+i \varepsilon_{i m}(\omega)=\varepsilon^{\prime}-i \varepsilon^{\prime \prime}$. We note that (1) can be written as $\dot{q}_{r f}=\omega \varepsilon^{\prime \prime} E_{R M S}^{2}$. As $\omega \rightarrow 0$ we approach a static field for which there is no dielectric loss and the permittivity approaches a constant denoted $\varepsilon_{0} \varepsilon_{s}$ where $\varepsilon_{s}$ is the static dielectric constant. As a result, we expect to see

$$
\begin{aligned}
& \varepsilon^{\prime \prime}(\omega) \rightarrow 0 \quad \text { as } \quad \omega \rightarrow 0 \\
& \varepsilon^{\prime}(\omega) \rightarrow \varepsilon_{0} \varepsilon_{s} \quad \text { as } \quad \omega \rightarrow 0
\end{aligned}
$$

Continuing our heuristic intuition-based arguments, we consider $\varepsilon^{\prime}$ and $\varepsilon^{\prime \prime}$ as $\omega \rightarrow \infty$. Clearly we do not want $\varepsilon^{\prime \prime}$ to become unbounded as $\omega$ becomes unbounded and so we require $\varepsilon^{\prime \prime}$ to approach some constant. However, if $\varepsilon^{\prime \prime}$ approaches a nonzero constant then $\dot{q}_{r f}$ will become unbounded as $\omega$ becomes unbounded, which is undesirable behavior since we expect a finite bound on $\dot{q}_{r f}$ for a given material. Hence, the limiting constant for $\varepsilon^{\prime \prime}$ must be zero or $\varepsilon^{\prime \prime} \rightarrow 0$ as $\omega \rightarrow \infty$. All materials have inertia and hence as $\omega \rightarrow \infty$, the dielectric material does not move and the RF electromagnetic signal must move at the speed of light. Therefore, by definition of $\varepsilon_{\infty}$ we expect

$$
\begin{aligned}
& \varepsilon^{\prime \prime}(\omega) \rightarrow 0 \quad \text { as } \omega \rightarrow \infty \\
& \varepsilon^{\prime}(\omega) \rightarrow \varepsilon_{0} \varepsilon_{\infty} \text { as } \omega \rightarrow \infty .
\end{aligned}
$$

Thus, if (1) is a correct expression we intuitively expect the limiting behavior for $\varepsilon^{\prime}, \varepsilon^{\prime \prime}$ given in (8), (9). As we shall see below, this can lead to inconsistencies.

A slightly different approach to required asymptotic behavior of dielectric parameters involves consideration of general polarization models in the frequency domain of the form

$$
\hat{P}(s)=\varepsilon_{0} \frac{p(s)}{q(s)} \hat{E}(s),
$$

where $\hat{P}$ and $\hat{E}$ are Laplace transforms of $P$ and $E$, respectively, and $p(s), q(s)$ are polynomials in $s$. Letting $s=i \omega$, from $D=\varepsilon_{0} \varepsilon_{\infty} E+P$ we have

$$
\begin{aligned}
\hat{D}(\omega) & =\left[\varepsilon_{0} \varepsilon_{\infty}+\frac{\varepsilon_{0} p(i \omega)}{q(i \omega)}\right] \hat{E}(\omega) \\
& =\left[\varepsilon_{0} \varepsilon_{\infty}+\frac{\varepsilon_{0} \overline{q(i \omega)} p(i \omega)}{|q(i \omega)|^{2}}\right] \hat{E}(\omega),
\end{aligned}
$$

where $\bar{z}$ and $|z|$ denote the usual complex conjugate and amplitude, respectively, of $z$. If

(degree of $p)<($ degree of $q)$ then $\hat{D} \rightarrow \varepsilon_{0} \varepsilon_{\infty} \hat{E}$ as $\omega \rightarrow \infty$ and we have what is sometimes defined as a stable material polarization model. We note that a model must be stable in order for it to satisfy (8) and (9). 


\subsubsection{Polarization assumptions}

In this section we describe polarization assumptions implicit in the derivation of Frohlich to obtain (1). There are two ways to view the polarization. The first is to write $P$ as a function of $E$ (this will result in (11) below), while the second is to write it as a function of both $E$ and $\dot{E}$ (this results in (12) below). We begin again with $D=\varepsilon_{0} \varepsilon_{\infty} E+P$ and recall that we are working with the periodic case in which $E=E_{0} \cos \omega t$ and $D=D_{0} \cos (\omega t-\delta)$. We have

$$
D_{0} \cos (\omega t-\delta)=\varepsilon_{0} \varepsilon_{\infty} E_{0} \cos \omega t+P
$$

or

$$
P=D_{0} \cos (\omega t-\delta)-\varepsilon_{0} \varepsilon_{\infty} E_{0} \cos \omega t
$$

We assume $D_{0}$ is proportional to $E_{0}$ (i.e., $D_{0}=\beta E_{0}$ where $\beta \neq 0$ may depend on $\omega$ ) and substitute into the above equation. In the case where we write $P$ as a function of $E$ we have

$$
\begin{aligned}
P & =\beta E_{0} \cos (\omega t-\delta)-\varepsilon_{0} \varepsilon_{\infty} E_{0} \cos \omega t \\
& =\beta E_{0}(\cos \omega t \cos \delta+\sin \omega t \sin \delta)-\varepsilon_{0} \varepsilon_{\infty} E_{0} \cos \omega t \\
& =\beta E \cos \delta+\beta \sqrt{E_{0}^{2}-E_{0}^{2} \cos ^{2} \omega t} \sin \delta-\varepsilon_{0} \varepsilon_{\infty} E \\
& =\beta E \cos \delta+\beta \sqrt{E_{0}^{2}-E^{2}} \sin \delta-\varepsilon_{0} \varepsilon_{\infty} E \\
& =E\left(\beta \cos \delta-\varepsilon_{0} \varepsilon_{\infty}\right)+\beta \sqrt{E_{0}^{2}-E^{2}} \sin \delta .
\end{aligned}
$$

Thus we have

$$
P=E\left(\beta \cos \delta-\varepsilon_{0} \varepsilon_{\infty}\right)+\beta \sqrt{E_{0}^{2}-E^{2}} \sin \delta
$$

from which we immediately observe that $P(-E) \neq-P(E)$ unless $\delta=0$ or $\beta=0$. If $\delta=0$ (i.e., $D=\varepsilon E$ ), we have $P=E\left(\beta-\varepsilon_{0} \varepsilon_{\infty}\right)=\chi E$ which is the standard polarization law for a linear (sometimes also called ideal) medium [Reitz, et.al., 1993, p. 110] and no apparent paradoxes arise. However, if we reverse the field $E$, in a dipolar medium we must reverse the polarization $P$, which implies $P(-E)=-P(E)$ and $P=P(E)$ must be odd in $\mathrm{E}$. As we have already observed from (11), for $\delta \neq 0, \beta \neq 0$, this is not true of the polarization implicit in the Frohlich derivation of (1). Therefore (11) is not a reasonable or valid polarization constitutive relation for any dipolar material. 
On the other hand, if we begin with (10) and proceed in a slightly different manner, we also obtain inconsistencies. We have

$$
\begin{aligned}
P & =\beta E_{0}(\cos \omega t \cos \delta+\sin \omega t \sin \delta)-\varepsilon_{0} \varepsilon_{\infty} E_{0} \cos \omega t \\
& =E\left(\beta \cos \delta-\varepsilon_{0} \varepsilon_{\infty}\right)-\frac{\beta E_{0}}{\omega}\left(\frac{d}{d t} \cos \omega t\right) \sin \delta \\
& =E\left(\beta \cos \delta-\varepsilon_{0} \varepsilon_{\infty}\right)-\frac{\beta}{\omega}\left(\frac{d}{d t} E_{0} \cos \omega t\right) \sin \delta \\
& =E\left(\beta \cos \delta-\varepsilon_{0} \varepsilon_{\infty}\right)-\frac{\beta}{\omega} \frac{d E}{d t} \sin \delta .
\end{aligned}
$$

Thus this argument leads to

$$
P=a E+b \dot{E}
$$

where $a=\beta \cos \delta-\varepsilon_{0} \varepsilon_{\infty}$ and $b=-\frac{\beta}{\omega} \sin \delta$. The second potential inconsistency can be illustrated by studying the form of $\varepsilon$ implicit in (12). We take the Laplace transform of (12) to obtain

$$
\hat{P}=(a+b s) \hat{E}
$$

where $a=\beta \cos \delta-\varepsilon_{0} \varepsilon_{\infty}$ and $b=-\frac{\beta}{\omega} \sin \delta$. Hence

$$
\begin{aligned}
\hat{D}(\omega) & =\left(\varepsilon_{0} \varepsilon_{\infty}+a+i b \omega\right) \hat{E} \\
& =\left[\left(\varepsilon_{0} \varepsilon_{\infty}+a\right)+i(b \omega)\right] \hat{E} \\
& =\left[\left(\beta \cos \delta+i\left(-\frac{\beta \omega}{\omega} \sin \delta\right)\right] \hat{E}\right. \\
& =[\underbrace{\beta \cos \delta}_{\varepsilon^{\prime}}-i \underbrace{\beta \sin \delta}_{\varepsilon^{\prime \prime}}] \hat{E}
\end{aligned}
$$

which relates $\beta$ to the expressions for $\varepsilon^{\prime}$ and $\varepsilon^{\prime \prime}$ defined in Section 2.1. Recalling the expected asymptotic behavior of $\varepsilon^{\prime}$ and $\varepsilon^{\prime \prime}$ in Section 2.2 leads to inconsistencies for $\beta$ in light of (13). To see this, we note that in the Frohlich derivation it is not explicitly stated whether or not the phase shift $\delta$ is frequency dependent. From (3), it is unlikely but possible that $\tan \delta$ is independent of $\omega$ even though $\varepsilon^{\prime}$ and $\varepsilon^{\prime \prime}$ generally are not. If $\delta$ is not frequency dependent (i.e., $\delta \neq \delta(\omega)$ ) we see from (13) that in order for (8) to hold we must have simultaneously $\beta \rightarrow \frac{\varepsilon_{0} \varepsilon_{s}}{\cos \delta}$ and $\beta \rightarrow 0$, as $\omega \rightarrow 0$, which leads to a contradiction. A similar contradiction arises for (9). Thus if $\delta \neq \delta(\omega),(8)$ and (9) are violated. In fact, $\delta$ should depend on frequency for a number of reasons, among them that the delay time should increase as frequency increases. If we assume 
$\delta=\delta(\omega)$ then the asymptotic behavior must be of a certain form in order to prevent apparent contradictions. This will be detailed below.

In a number of cases, permittivities based on other polarization models do meet the requirements of (8) and (9). We illustrate this with examples involving two common polarization models. We first consider the Debye model [Reitz, et.al., 1993, p. 514]:

$$
\dot{P}+\frac{1}{\tau} P=\frac{\varepsilon_{0}\left(\varepsilon_{s}-\varepsilon_{\infty}\right)}{\tau} E
$$

where $\tau$ is the relaxation time, $\varepsilon_{s}$ is the static dielectric constant, and $\varepsilon_{\infty}$ is the limiting value of the permittivity as the frequency becomes unbounded. We take the Laplace transform to obtain

$$
\left(s+\frac{1}{\tau}\right) \hat{P}=\frac{\varepsilon_{0}\left(\varepsilon_{s}-\varepsilon_{\infty}\right)}{\tau} \hat{E}
$$

Hence

$$
\hat{D}=\left(\varepsilon_{0} \varepsilon_{\infty}+\frac{\varepsilon_{0}\left(\varepsilon_{s}-\varepsilon_{\infty}\right)}{1+\tau s}\right) \hat{E} .
$$

Taking $s=i \omega$ we have

$$
\begin{aligned}
\hat{D}(\omega) & =\left[\varepsilon_{0} \varepsilon_{\infty}+\frac{\varepsilon_{0}\left(\varepsilon_{s}-\varepsilon_{\infty}\right)}{1+i \omega \tau}\right] \hat{E} \\
& =\left[\varepsilon_{0} \varepsilon_{\infty}+\frac{\varepsilon_{0}\left(\varepsilon_{s}-\varepsilon_{\infty}\right)(1-i \omega \tau)}{1+\omega^{2} \tau^{2}}\right] \hat{E} \\
& =[\underbrace{\left(\varepsilon_{0} \varepsilon_{\infty}+\frac{\varepsilon_{0}\left(\varepsilon_{s}-\varepsilon_{\infty}\right)}{1+\omega^{2} \tau^{2}}\right)}_{\varepsilon^{\prime}}-i \underbrace{\left(\frac{\varepsilon_{0} \omega \tau\left(\varepsilon_{s}-\varepsilon_{\infty}\right)}{1+\omega^{2} \tau^{2}}\right)}_{\varepsilon^{\prime \prime}}] \hat{E} .
\end{aligned}
$$

As $\omega \rightarrow 0$, it is clear that $\varepsilon^{\prime} \rightarrow \varepsilon_{0} \varepsilon_{s}$ and $\varepsilon^{\prime \prime} \rightarrow 0$. As $\omega$ becomes unbounded we see that $\varepsilon^{\prime} \rightarrow \varepsilon_{0} \varepsilon_{\infty}$ and $\varepsilon^{\prime \prime} \rightarrow 0$, which is exactly the behavior required by (8) and (9).

We next consider the Lorentz model which is described in detail in [Reitz, et.al., 1993, pp. 495-499] given by

$$
\ddot{P}+\frac{1}{\tau} \dot{P}+\omega_{0}^{2} P=\varepsilon_{0} \omega_{p}^{2} E
$$

Here $\tau$ is again the relaxation time, $\omega_{0}$ is the natural frequency of the undamped oscillator, and $\omega_{p}=N e / \varepsilon_{0} m$ where $N$ is the number of charges per unit volume, $e$ is the displaced charge and $m$ is mass of the particle in the damped harmonic oscillator paradigm that is the basis of the Lorentz model. Again, 
we take the Laplace transform and obtain

$$
s^{2} \hat{P}+\frac{1}{\tau} s \hat{P}+\omega_{0}^{2} \hat{P}=\varepsilon_{0} \omega_{p}^{2} \hat{E}
$$

and taking $s=i \omega$ we have

$$
\hat{P}=\left[\frac{\varepsilon_{0} \tau \omega_{p}^{2}}{\tau\left(\omega_{0}^{2}-\omega^{2}\right)+i \omega}\right] \hat{E} .
$$

Using again $D=\varepsilon_{0} \varepsilon_{\infty} E+P$ we find

$$
\begin{aligned}
\hat{D} & =\left[\varepsilon_{0} \varepsilon_{\infty}+\frac{\varepsilon_{0} \tau \omega_{p}^{2}}{\tau\left(\omega_{0}^{2}-\omega^{2}\right)+i \omega}\right] \hat{E} \\
& =\left[\varepsilon_{0} \varepsilon_{\infty}+\frac{\varepsilon_{0}\left(\tau\left(\omega_{0}^{2}-\omega^{2}\right)-i \omega\right) \tau \omega_{p}^{2}}{\tau^{2}\left(\omega_{0}^{2}-\omega^{2}\right)^{2}+\omega^{2}}\right] \hat{E} \\
& =[\underbrace{\left(\varepsilon_{0} \varepsilon_{\infty}+\frac{\varepsilon_{0} \tau^{2}\left(\omega_{0}^{2}-\omega^{2}\right) \omega_{p}^{2}}{\tau^{2}\left(\omega_{0}^{2}-\omega^{2}\right)^{2}+\omega^{2}}\right)}_{\varepsilon^{\prime}}-i \underbrace{\left(\varepsilon_{0} \frac{\varepsilon_{0} \omega \tau \omega_{p}^{2}}{\tau^{2}\left(\omega_{0}^{2}-\omega^{2}\right)^{2}+\omega^{2}}\right)}_{\varepsilon^{\prime \prime}}] \hat{E} .
\end{aligned}
$$

As $\omega \rightarrow \infty$ we see that $\varepsilon^{\prime} \rightarrow \varepsilon_{0} \varepsilon_{\infty}$ and $\varepsilon^{\prime \prime} \rightarrow 0$. Additionally, as $\omega \rightarrow 0, \varepsilon^{\prime \prime} \rightarrow 0$, but

$$
\varepsilon^{\prime}=\varepsilon_{0} \varepsilon_{\infty}+\frac{\varepsilon_{0} \tau^{2} \omega_{0}^{2} \omega_{p}^{2}}{\tau^{2} \omega_{0}^{4}}
$$

and it is not clear that this is equal to $\varepsilon_{0} \varepsilon_{s}$. However, if we let $\omega_{p}^{2}=\omega_{0}^{2}\left(\varepsilon_{s}-\varepsilon_{\infty}\right)$ [Scaife, 1989, p. 59], then $\varepsilon^{\prime} \rightarrow \varepsilon_{0} \varepsilon_{s}$ as required in (8) and the Lorentz model satisfies (8) and (9).

We turn our attention to the form of $\dot{q}_{r f}$ in (1). Clearly $\dot{q}_{r f} \rightarrow 0$ as $\omega \rightarrow 0$ since we know $\varepsilon^{\prime \prime} \rightarrow 0$ as $\omega \rightarrow 0$. As $\omega$ becomes unbounded, however, the behavior of $\dot{q}_{r f}$ is not as clear. Since we want $\dot{q}_{r f}$ to approach some constant as $\omega$ becomes unbounded, we want $\varepsilon^{\prime \prime} \rightarrow 0$ faster than $\omega \rightarrow \infty$. In order for this to hold, $\varepsilon^{\prime \prime}$ must behave like $\frac{1}{\omega^{1+\alpha}}$ for some $\alpha \geq 0$. Similarly, $\tan \delta$ must behave like $\frac{1}{\omega^{1+\alpha}}$. If this is true, as $\omega$ becomes unbounded $\dot{q}_{r f}$ will behave like $\frac{1}{\omega^{\alpha}}$ which approaches 0 for $\alpha>0$ and approaches a constant for $\alpha=0$. If this is not true, $\dot{q}_{r f}$ will become unbounded as $\omega$ becomes unbounded, and we have an objection to $(1)$.

We note that stability is not enough to guarantee that $\dot{q}_{r f}$ will approach some constant as $\omega$ becomes unbounded. Stability guarantees only that $\varepsilon^{\prime \prime}$ approaches 0 , giving no information on the rate at which $\varepsilon^{\prime \prime}$ approaches 0 relative to the rate at which $\omega$ becomes unbounded. Therefore, we have a stronger restriction on the materials whose behavior can be approximated by (1). In Section 3 we derive alternatives to (1) which satisfy this added restriction. 


\subsection{Alternate derivation of standard RF term}

Since we encounter difficulties with the implicit polarization assumption in the derivation in Section 2.1, we present a derivation of (1) with arguments that do not involve the polarization. This derivation, based on the report [Lord, 1983] and arguments in [White, 1973], relies on Joule's heating law along with a number of other somewhat questionable tacit assumptions. Again one obtains the standard RF heating rate term (1), but from energy considerations. Joule's heating law [Elliot, 1993, p. 489], under assumptions of no conduction, radiation, or other losses, i.e., $100 \%$ efficiency, is given by

$$
\frac{d U}{d t}=\sigma E^{2}
$$

where $U$ is internal energy, $\sigma$ represents conductivity, and $E$ represents the instantaneous electric field in the dielectric. We note that the dielectric must satisfy Ohm's Law $(J=\sigma E$, a standard assumption for linear isotropic materials [Reitz, et.al., 1993, p. 167]) in order for this form of Joule's law to be valid. From (16) we have over any time period $\left[t_{0}, t\right]$

$$
\int_{t_{0}}^{t} \frac{d U}{d t}=\int_{t_{0}}^{t} \sigma E^{2} d t
$$

or, assuming $\sigma$ constant,

$$
\Delta U=\sigma \int_{t_{0}}^{t} E^{2} d t \quad \text { where } \Delta U=U(t)-U\left(t_{0}\right) .
$$

If we further assume a periodic field $E=E_{0} \cos \omega t$, then we have

$$
\begin{aligned}
\Delta U & =\sigma E_{0}^{2} \int_{t_{0}}^{t} \cos ^{2} \omega s d s \\
& =\sigma E_{0}^{2} \int_{t_{0}}^{t} \frac{1}{2}[1+\cos 2 \omega s] d s \\
& =\sigma E_{0}^{2}\left[\frac{1}{2} s+\frac{1}{4 \omega} \sin 2 \omega s\right]_{t_{0}}^{t} \\
& =\sigma E_{0}^{2}(\frac{1}{2}\left(t-t_{0}\right)+\underbrace{\frac{1}{4 \omega} \sin 2 \omega t}_{\approx 0}-\underbrace{\frac{1}{4 \omega} \sin 2 \omega t_{0}}_{\approx 0})
\end{aligned}
$$

where the second and third terms in the last expression are very small (and hence neglected) because RF heating involves $\omega$ of approximately $10^{6}-10^{7} \mathrm{~Hz}$. If we assume $t_{0}=0$, we have

$$
\Delta U=\frac{1}{2} \sigma E_{0}^{2} t
$$


We next consider the conductivity $\sigma$ and argue that it is given by $\sigma=\omega \varepsilon_{0} \varepsilon_{R}^{\prime} \tan \delta$. We note that if (1) is to hold, this must be the case.

The derivation is suggested by White [1973] who notes that in the literature conductivity is commonly written $\sigma=\omega \varepsilon^{\prime \prime}$. Using this along with the definition of $\varepsilon^{\prime \prime}$ given in Section 2.1 we see that

$$
\begin{aligned}
\sigma & =\omega \varepsilon^{\prime} \tan \delta \\
& =\omega \varepsilon_{0} \frac{\varepsilon^{\prime}}{\varepsilon_{0}} \tan \delta \\
& =\omega \varepsilon_{0} \varepsilon_{R}^{\prime} \tan \delta .
\end{aligned}
$$

With this form for $\sigma$ and the expression for $\frac{\Delta U}{t}$, we see that the rate of absorption $L$ can be written

$$
L=\frac{\Delta U}{t}=\frac{1}{2} \sigma E_{0}^{2}=\frac{1}{2} \omega \varepsilon_{0} \varepsilon_{R}^{\prime} E_{0}^{2} \tan \delta .
$$

Setting $E_{R M S}=E_{0} / \sqrt{2}$, we obtain

$$
\dot{q}=L=\omega \varepsilon_{0} \varepsilon_{R}^{\prime} E_{R M S}^{2} \tan \delta
$$

We see that this derivation yields exactly the same expression as the Frohlich derivation. Although this derivation does not depend explicitly on the polarization assumptions found in our earlier derivation, they are inherent in our definitions of $\sigma$ and therefore this derivation leads to the same conceptual difficulties as the previous one. Moreover, the assumptions (implicit as well as explicit) inherent in the arguments (e.g., constant conductivity, ohmic rather than dielectric heating, etc.) really are not viable for most dielectrics.

\section{Alternate RF expressions}

At this point we briefly summarize the difference between ionic and dipolar conduction as presented in [White, 1973]. In general adhesives, ions naturally exist. Concentration is dependent on chemistry, presence of water and other impurities or contamination. These ions are electrically charged and as a result are attracted or repulsed by electric fields. The ions then collide many times (depending on the number of random ions, density of material and the frequency of the field) with other un-ionized molecules which provides a two step conversion process in which electric field energy is converted into ordered kinetic energy and then into disordered kinetic energy where it is appropriate to regard it as heat. Dipolar conduction, on 
the other hand takes place due to the dipolar rotation of the molecules in the material. Here there is a conversion of energy from electric field energy to potential energy as the electric field builds up and then to stored random kinetic or thermal energy in the material as the field dies down [White, 1973, p. 47]. We feel that the epoxy system which we are modeling has very low ionic content. Hence, for our derivations below we will assume all heating is dipolar, but in reality, depending on the material, there may be other ionic contributions.

Since we have shown that different derivations of (1) depend implicitly on a polarization model with some inconsistencies, we now derive alternate forms of the $\dot{q}_{r f}$ expression based on the Debye and Lorentz polarization models often assumed in the electromagnetic materials literature. We derive first a Debye-based heating expression and discuss its asymptotic behavior and then do the same with a Lorentz-based expression. We do this to illustrate the considerations that must be incorporated into any serious effort to derive general RF expressions.

\subsection{Derivation of Debye-based RF expression}

We wish to derive an RF heating term based on a Debye polarization model. Recall (see Section 2.2.1) that the Debye model is represented by the equation

$$
\dot{P}+\frac{1}{\tau} P=\frac{\varepsilon_{0}\left(\varepsilon_{s}-\varepsilon_{\infty}\right)}{\tau} E
$$

If we let $a=\frac{1}{\tau}$ and $b=\frac{\varepsilon_{0}\left(\varepsilon_{s}-\varepsilon_{\infty}\right)}{\tau}$, we have

$$
\dot{P}+a P=b E
$$

Again we assume $E=E_{0} \cos \omega t$ where $\omega / 2 \pi$ is the frequency in cycles per second. Substituting into (18) we obtain

$$
\dot{P}+a P=b E_{0} \cos \omega t
$$

which yields the solution

$$
P(t)=P_{0} e^{-a t}+b E_{0} e^{-a t} \int_{0}^{t} e^{a s} \cos \omega s d s .
$$

Substituting (19) into the equation $D=\varepsilon_{0} \varepsilon_{\infty} E+P$, we find

$$
D=\varepsilon_{0} \varepsilon_{\infty} E_{0} \cos \omega t+P_{0} e^{-a t}+b E_{0} e^{-a t} \int_{0}^{t} e^{a s} \cos \omega s d s
$$


As in the previous derivations (see (5)) we have

$$
L=\frac{\omega}{2 \pi} \int_{0}^{2 \pi / \omega} E \frac{\partial D}{\partial t} d t
$$

So from (20) we obtain

$$
\begin{aligned}
L & =\frac{\omega}{2 \pi} \int_{0}^{2 \pi / \omega} E_{0} \cos \omega t \frac{\partial}{\partial t}\left[\varepsilon_{0} \varepsilon_{\infty} E_{0} \cos \omega t+P_{0} e^{-a t}+b E_{0} e^{-a t} \int_{0}^{t} e^{a s} \cos \omega s d s\right] d t \\
& =\frac{\omega}{2 \pi} E_{0}^{2} \int_{0}^{2 \pi / \omega} \frac{\varepsilon_{0} \varepsilon_{\infty}}{2} \frac{\partial}{\partial t}\left(\cos ^{2} \omega t\right)+\frac{P_{0}}{E_{0}} \cos \omega t \frac{\partial}{\partial t}\left(e^{-a t}\right)+b \cos \omega t \frac{\partial}{\partial t}\left[e^{-a t} \int_{0}^{t} e^{a s} \cos \omega s d s\right] d t \\
& =\frac{\omega}{2 \pi} E_{0}^{2}\left[-\frac{a P_{0}}{E_{0}} \int_{0}^{2 \pi / \omega} e^{-a t} \cos \omega t d t+b \int_{0}^{2 \pi / \omega} \cos \omega t \frac{\partial}{\partial t} \int_{0}^{t} e^{a s} \cos \omega s d s d t\right] .
\end{aligned}
$$

Carrying out the remaining integration, we find

$$
L=\frac{\omega}{2 \pi} E_{0}^{2}\left[\frac{a^{2} P_{0}}{E_{0}} \frac{\left(e^{-2 \pi a / \omega}-1\right)}{a^{2}+\omega^{2}}+\frac{b\left(\omega \pi\left(a^{2}+\omega^{2}\right)-a^{3} e^{-2 \pi a / \omega}+a^{3}\right)}{\left(a^{2}+\omega^{2}\right)^{2}}\right]
$$

and if we let $E_{R M S}=\frac{E_{0}}{\sqrt{2}}$, we have an alternate RF term:

$$
\dot{q}=L=\omega\left[\frac{a^{2} P_{0}}{\pi E_{0}} \frac{\left(e^{-2 \pi a / \omega}-1\right)}{a^{2}+\omega^{2}}+\frac{b\left(\omega \pi\left(a^{2}+\omega^{2}\right)-a^{3} e^{-2 \pi a / \omega}+a^{3}\right)}{\pi\left(a^{2}+\omega^{2}\right)^{2}}\right] E_{R M S}^{2}
$$

where $a=\frac{1}{\tau}$ and $b=\frac{\varepsilon_{0}\left(\varepsilon_{s}-\varepsilon_{\infty}\right)}{\tau}$.

We study the behavior of this term which can be rewritten

$$
\dot{q}=\omega \Gamma E_{R M S}^{2}
$$

where $\Gamma$ represents the bracketed term in equation (21). This has the same form as (1), but the constants must be interpreted differently (in (1) $\Gamma$ is given by $\varepsilon_{0} \varepsilon_{R}^{\prime} \tan \delta$ ). It is easily seen that as $\omega$ approaches 0 , $\Gamma \rightarrow \frac{1}{\pi}\left(-\frac{P_{0}}{E_{0}}+\varepsilon_{0}\left(\varepsilon_{s}-\varepsilon_{\infty}\right)\right)$, which is constant and hence $\dot{q} \rightarrow 0$, which is the same behavior found for (1). As $\omega$ approaches infinity, however, we see much different behavior. We expand $\Gamma$ in (21) to obtain

$$
\begin{aligned}
\Gamma & =\frac{a^{2} P_{0}}{\pi E_{0}}[\overbrace{\frac{e^{-2 \pi a / \omega}}{a^{2}+\omega^{2}}}^{(a)}-\overbrace{\frac{1}{a^{2}+\omega^{2}}}^{(b)}]+\overbrace{\frac{b \omega a^{2}}{\left(a^{2}+\omega^{2}\right)^{2}}}^{(c)} \\
+ & \underbrace{\frac{b \omega^{3}}{\left(a^{2}+\omega^{2}\right)^{2}}}_{(d)}-\underbrace{\frac{b a^{3} e^{-2 \pi a / \omega}}{\pi\left(a^{2}+\omega^{2}\right)^{2}}}_{(e)}+\underbrace{\frac{a^{3}}{\pi\left(a^{2}+\omega^{2}\right)^{2}}}_{(f)} .
\end{aligned}
$$

Clearly terms (a), (b), (e), and (f) go to 0 as $\omega \rightarrow \infty$, and by applying L'Hôpital's rule to the remaining terms we see that they too approach 0 as $\omega$ approaches infinity. If we relate $\Gamma$ to $\varepsilon^{\prime \prime}$ in the standard term 
and recall our discussion of the desired behavior of $\varepsilon^{\prime \prime}$ in Section 2.2 .1 where we let $\varepsilon_{i m}=\varepsilon^{\prime \prime}$, this is exactly what we expect to see. If we now look at the entire term given in (21), using the same methods for taking the limits as above, we find that as $\omega \rightarrow \infty$,

$$
\dot{q} \rightarrow b=\frac{\varepsilon_{0}\left(\varepsilon_{s}-\varepsilon_{\infty}\right)}{\tau} .
$$

That is, as the frequency becomes very large, the rate of heating will approach a finite constant value, as discussed in Section 2.2.1.

\subsection{Derivation of Lorentz-based RF expression}

Another common polarization model is the Lorentz model. Following the same steps used Section 3.1, we derive an RF term based on this model. Recall that the Lorentz model is represented by the equation

$$
\ddot{P}+\frac{1}{\tau} \dot{P}+\omega_{0}^{2} P=\varepsilon_{0} \omega_{p}^{2} E
$$

If we let $a=\frac{1}{\tau}, b=\omega_{0}$, and $c=\varepsilon_{0} \omega_{p}^{2}$, we have

$$
\ddot{P}+a \dot{P}+b^{2} P=c E \text {. }
$$

We assume $E=E_{0} \cos \omega t$ and substitute into (23) to obtain

$$
\ddot{P}+a \dot{P}+b^{2} P=c E_{0} \cos \omega t
$$

After applying the variation of constants formula, we find a solution $P$ to (23)

$$
\begin{aligned}
P(t) & =\frac{P_{0}\left(e^{\lambda_{1} t} \lambda_{2}-e^{\lambda_{2} t} \lambda_{1}\right)}{\gamma}+\frac{P_{1}\left(e^{\lambda_{2} t}-e^{\lambda_{1} t}\right)}{\gamma} \\
& +\frac{c E_{0}}{\gamma}\left[\frac{\omega \sin \omega t+\lambda_{2} e^{\lambda_{2} t}-\lambda_{2} \cos \omega t}{\lambda_{2}^{2}+\omega^{2}}+\frac{\lambda_{1} \cos \omega t-\omega \sin \omega t-\lambda_{1} e^{\lambda_{1} t}}{\lambda_{1}^{2}+\omega^{2}}\right]
\end{aligned}
$$

where $\gamma=\sqrt{a^{2}-4 b^{2}}, \lambda_{1}=\frac{-a-\gamma}{2}$, and $\lambda_{2}=\frac{-a+\gamma}{2}$. We note that $\lambda_{1}$ and $\lambda_{2}$ are the eigenvalues of the system of first order equations equivalent to (23) (which is a damped harmonic oscillator) and since this represents an asymptotically stable dynamical system [Brauer and Nohel, 1969, p. 151], we have $\operatorname{Re}\left(\lambda_{i}\right)<0$, for $i=1,2$. Following the previous arguments, we wish to find $L$ given by

$$
L=\frac{\omega}{2 \pi} \int_{0}^{2 \pi / \omega} E \frac{\partial D}{\partial t} d t
$$


where $D=\varepsilon_{0} \varepsilon_{\infty} E+P$ and $P$ is given by (24). After some simple integration, this reduces to

$$
L=\frac{\omega E_{0}}{2 \pi} \int_{0}^{2 \pi / \omega} \cos \omega t \frac{\partial P}{\partial t} d t
$$

Carrying out the remaining integration and letting $E_{R M S}=E_{0} / \sqrt{2}$, after some straightforward but tedious calculations, we obtain a form for the RF term:

$$
\begin{aligned}
\dot{q}=L & =\omega\left[\frac { 1 } { \pi \gamma } \left(\left(\frac{\lambda_{1} \lambda_{2} P_{0}}{E_{0}}-\frac{\lambda_{1} P_{1}}{E_{0}}-\frac{c \lambda_{1}^{2}}{\left(\lambda_{1}^{2}+\omega^{2}\right)}\right) \frac{\lambda_{1}\left(e^{2 \pi \lambda_{1} / \omega}-1\right)}{\left(\lambda_{1}^{2}+\omega^{2}\right)}\right.\right. \\
& +\left(\frac{-\lambda_{1} \lambda_{2} P_{0}}{E_{0}}+\frac{\lambda_{2} P_{1}}{E_{0}}+\frac{c \lambda_{2}^{2}}{\left(\lambda_{2}^{2}+\omega^{2}\right)}\right) \frac{\lambda_{2}\left(e^{2 \pi \lambda_{2} / \omega}-1\right)}{\left(\lambda_{2}^{2}+\omega^{2}\right)} \\
& \left.\left.+c \pi \omega\left(\frac{1}{\lambda_{2}^{2}+w^{2}}-\frac{1}{\lambda_{1}^{2}+\omega^{2}}\right)\right)\right] E_{R M S}^{2} .
\end{aligned}
$$

We investigate the behavior of this expression, which can again be written in the form

$$
\dot{q}=\omega \Gamma E_{R M S}^{2}
$$

where $\Gamma$ is the bracketed term in (25). We first consider the behavior as $\omega \rightarrow 0$. We note that as $\omega$ approaches $0, e^{2 \pi \lambda_{1} / \omega} \rightarrow 0$ since, as stated previously, $\operatorname{Re}\left(\lambda_{1}\right)$ is less than 0 . The same holds for the exponential involving $\lambda_{2}$. From this, it is clear that as $\omega \rightarrow 0$,

$$
\Gamma \rightarrow \frac{1}{\pi \gamma}\left[\left(-\frac{\lambda_{1} \lambda_{2} P_{0}-\lambda_{1} P_{1}}{E_{0}}+c\right) \frac{1}{\lambda_{1}}+\left(-\frac{-\lambda_{1} \lambda_{2} P_{0}+\lambda_{2} P_{1}}{E_{0}}-c\right) \frac{1}{\lambda_{2}}\right]
$$

which is constant and again we see that $\dot{q} \rightarrow 0$ as $\omega \rightarrow 0$. Applying the same arguments as in Section 3.1 , it is easy to see that $\dot{q} \rightarrow 0$ as $\omega \rightarrow \infty$ which is the same behavior found in the Debye-based term. To see this note that the terms

$$
\frac{c \pi \omega^{2}}{\lambda_{2}^{2}+\omega^{2}} \quad \text { and } \quad \frac{c \pi \omega^{2}}{\lambda_{1}^{2}+\omega^{2}}
$$

each approach $c \pi$ as $\omega \rightarrow \infty$ while all of the other terms approach 0 , so we find $\dot{q} \rightarrow(c \pi-c \pi) E_{R M S}=0$ as $\omega \rightarrow \infty$. Again, this limiting behavior is more intuitively satisfying than the behavior of the standard RF term given by (1).

Most dielectric materials are quite complicated in their polarization mechanisms and the related constitutive laws usually cannot be expressed simply in terms of a simple Debye or Lorentz law. However, it is quite common to model polarization using combinations of multiples of these (and other) simple polarization relationships [Albanese, 1997], [Oughstun, et.al., 1993]. Adhesives are no less complex with 
respect to polarization. Almost all adhesives (two component epoxies, water based urethanes as well as more general two component urethanes, acrylics, etc.) involve liquid as well as solid components. Moreover, during the adhesive curing process, transitions from liquid to solid phases occur, further complicating the derivation of any polarization based RF heating law. Thus while the above examples illustrate how one might go about deriving an RF term from a given polarization law and checking it for consistency, it is most unlikely that either of the specific examples (Debye or Lorentz), used above for illustrative purposes, will provide an adequate basis for deriving RF terms for many adhesives used in practice. Our current research efforts (both theoretical and experimental) are focused precisely on this topic.

\section{Summary and implications}

We have derived several expressions for the term $\dot{q}_{r f}$. We see that all of these expressions are of the form $\dot{q}_{r f}=\omega \Gamma E_{R M S}^{2}$ where the definition of $\Gamma$ depends on the polarization model used in the derivation. More importantly, we have shown that the standard term (1) widely found in the electromagnetic heating literature corresponds to a polarization model with inherent inconsistencies. We have thus demonstrated the need for alternate heating expressions. We follow the same steps used in the usual derivation to derive alternate expressions based on two well known polarization models - Debye and Lorentz. Based on our analysis, we suggest that while the Debye and Lorentz models are too simple to approximate the polarization behavior of most adhesives, they at least result in RF heating terms that possess desired asymptotic behavior properties lacking in the standard RF expression found in the literature. Moreover, these examples illustrate an approach that can be followed to derive RF heating expressions based on a given polarization model.

Arguments for the use of (1) due to the simple fact that $\varepsilon^{\prime}$ and $\varepsilon^{\prime \prime}$ can be found easily through experimentation are not persuasive since the expression is not based on physically realistic material assumptions. We can relate $\varepsilon^{\prime}$ and $\varepsilon^{\prime \prime}$ to the Debye model using the form of (14) and to the Lorentz model using (15). For example, if we know something about $\tau$ in the Debye model, we can use these relationships to determine $\varepsilon_{s}$

and $\varepsilon_{\infty}$ and hence obtain our equation (21) in terms of $\varepsilon^{\prime}$ and $\tan \delta$. Similarly, if we have information about two of the parameters in the Lorentz medium, we can write (25) in a similar form. Therefore, we see that 
not only do these terms model the desired asymptotic behavior of RF heating more accurately, but with some information about one or two of the terms, they can be estimated using the same measured quantities used in the standard RF term (1). There is, of course, a remaining challenge in deriving RF heating terms based on more realistic polarization assumptions for adhesives while at the same time obtaining RF terms parameterized in a manner consistent with the reliable estimation of parameters from experimental data. 


\section{References}

[Albanese, 1997] R.A. Albanese, Wave propagation inverse problems in medicine and environmental health, Proc. of the IMA Conference on Waves and Scattering, Springer Verlag, 1997, 1-11.

[Banks, et. al., 1998] H.T. Banks, S.R. Durso, M.A. Goodhart, and K. Ito, Nonlinear exothermic contributions to radio-frequency bonding of adhesives, Tech. Rep. CRSC-TR98-24, NCSU, June, 1998.

[Bejan, 1993] A. Bejan, Heat Transfer, John Wiley \& Sons, New York, 1993.

[Brauer and Nohel, 1969] F. Brauer and J.A. Nohel, The Qualitative Theory of Ordinary Differential Equations, Dover Publications, New York, 1969.

[Bunget and Popescu, 1984] I. Bunget and M. Popescu, Physics of Solid Dielectrics, Elsevier, New York, 1984.

[Burden and Faires, 1993] R.L. Burden and J.D. Faires, Numerical Analysis, PWS Publishing, Boston, 1993.

[Elliot, 1993] R.S. Elliot, Electromagnetics: History, Theory, and Applications, IEEE Press, New York, 1993.

[Frohlich, 1958] H. Frohlich, Theory of Dielectrics: Dielectric Constant and Dielectric Loss, Clarendon Press, Oxford, 1958.

[Lord, 1983] Lord Corporation-Technical Report: Microwave Heating of Dielectric Adhesives, 1983.

[Malaczynski, 1988] G.W. Malaczynski, Adhesive processing by electromagnetic irradiation, Polymer Engineering and Science, 28 (1988), 1270-1274.

[Malaczynski and Cipinski, 1996] G.W. Malaczynski and G.J. Cinpinski, Selection of adhesive system for radio-frequency heating of structural sheet molding compound components, Polymer Engineering and Science, 36 (1996), 106-116.

[Oughstun, et.al., 1993] Oughstun, K.E., Laurens, J.E.K., and Balictsis, C.M., Asymptotic description of electromagnetic pulse propagation in a linear dispersive medium, in Ultra-Wideband, Short-Pulse Electromagnetics (ed. by Bertoni, H.L. et.al.), Plenum Press, New York, i1993, 223-250. 
[Reitz, et.al., 1993] J. R. Reitz, F.J. Milford, and R.W. Christy, Foundations of Electromagnetic Theory, Addison-Wesley, New York, 1993.

[Ross, 1982] D. Ross, Dielectric heating, Journal of Applied Physics, 53 (1982), 5823-5827.

[Scaife, 1989] B. K.P. Scaife, Principles of Dielectrics, Clarendon Press, Oxford, 1989.

[Stuerga and Gaillard, 1996] D.A.C. Stuerga and P. Gaillard, Microwave athermal effects in chemistry: A myth's autopsy. Part I: Historical background and fundamentals of wave-matter interaction, Journal of Microwave Power and Engineering, 31 (1996), 87-99.

[White, 1973] J. R. White, Why materials heat, Trans. IMPI, 1 (1973),40-65. 